\title{
Understanding the role of echocardiography in remodeling after acute myocardial infarction and development of heart failure with preserved ejection fraction
}

\author{
Raluca Tomoaia ${ }^{1,2}$, Ruxandra Stefana Beyer ${ }^{1}$, Gelu Simu ${ }^{2,3}$, Adela Mihaela Serban ${ }^{1,2}$, Dana \\ Pop $^{2,3}$
}

${ }^{1}$ Heart Institute "N. Stancioiu”, 2 "Iuliu Hatieganu” University of Medicine and Pharmacy, ${ }^{3}$ Cardiology Department, Rehabilitation Hospital, Cluj-Napoca, Romania

\begin{abstract}
Despite the use of reperfusion therapies in the last decades, acute myocardial infarction further remains one of the most frequent causes of mortality. This is mainly caused by changes in the ventricular architecture leading to ventricular remodeling, followed by progressive development of heart failure. Transthoracic echocardiography is a non-invasive instrument which can provide information about the extent of the ischemic process and its consequences but can also predict the outcomes after myocardial infarction. Although standard echocardiographic parameters are currently used for risk stratification of these patients, they might not truly reflect left ventricular systolic dysfunction in acute myocardial infarct patients, since the detection of subtle changes in the myocardial function is beyond their limits. The aim of this review is to underline the use of advanced echocardiographic parameters in identifying patients at risk for developing post-acute myocardial infarction heart failure and subsequent adverse events. Advanced echocardiographic parameters derived from speckle tracking echocardiography provide a detailed assessment on the global and regional left ventricular deformation. Therefore, speckle tracking echocardiography has a major role in predicting the prognosis of acute myocardial infarction patients and particularly in the development of subsequent heart failure, which might be prevented with early initiation of adequate therapy.
\end{abstract}

Keywords: myocardial infarction; heart failure; speckle tracking; echocardiography

\section{Introduction}

Ischemic heart disease is at the present the most frequent cause of death worldwide. The incidence of mortality caused by acute myocardial infarction (AMI) has decreased in the last 30 years due to the use of reperfusion therapies [primary percutaneous coronary intervention (PCI) or thrombolysis], associated with antithrom-

Received 22.10.2018 Accepted 04.12.2018

Med Ultrason

2019, Vol. 21, No 1, 69-76

Corresponding author: Raluca Tomoaia

Heart Institute "N. Stancioiu",

"Iuliu Hatieganu" University of Medicine and

Pharmacy, 17-19/18 Ploiești Street,

400157 Cluj-Napoca, Romania

Phone: 0040751350057

E-mail: raluca.tomoaia@gmail.com botic treatment and secondary prevention. Nevertheless, mortality still remains high (one-years mortality in patients with ST-elevation acute myocardial infarction is approximately $10 \%$ ), because of other factors that influence the prognostic of this pathology [1]. These factors can lead to post-AMI remodeling of the ventricular fibers. Ventricular remodeling refers to the alteration of ventricular structure, due to the progressive changes in its geometry (modifications in wall thickness, cavity diameter and progression from elliptical to spherical configuration). Potential factors involved in this process are myocytic death, imbalance between oxygen supply and consumption, oxidative stress, inflammation, fibrosis and neuro-hormonal activation [2]. All of these mechanisms lead to a deterioration of the ventricular function, with progressive development of heart failure (HF).

Although the incidence HF has decreased over the last few years due to the reperfusion therapies, mortality 
seems to be associated with initial left ventricular (LV) systolic dysfunction [3]. Furthermore, the number of patients developing diastolic dysfunction still remains high (20-30\%) [4]. Progression of diastolic dysfunction to HF with preserved ejection fraction (HFpEF) is associated with a poor prognosis and increase in the incidence of adverse effects due to the therapeutic limitations [5].

Post-AMI systolic LV dysfunction in relation to the development of HF has been intensively studied, but data relating to the prevalence and prognosis of patients with post-AMI HFpEF are still lacking [6,7]. A few studies specifically evaluated post-AMI HFpEF, but generally without simultaneous assessment of both ST-segment elevation MI (STEMI) and non-ST-segment elevation MI (NSTEMI). Moreover, heterogeneous LVEF cut-off points were used in the establishment of the diagnosis of HFpEF [8-10].

Studies using various imaging techniques (cardiac magnetic resonance, echocardiography, radionuclide ventriculography) in the evaluation of post-AMI ventricular remodeling have demonstrated that initial LV volumes and their modification on follow-up have prognostic implications. Transthoracic echocardiography (TTE) is a noninvasive investigation, which can be safely and routinely used. Among systolic function parameters, LV ejection fraction (LVEF), LV end diastolic volume index (LVEDDVi), LV end systolic volume index (LVESVi), LV mass index (LMVi), end-diastolic wall thickness (EDWT), end systolic wall thickness (ESWT) and the wall motion index (WMI) have proven to be of prognostic value [11]. Although LVEF is easy to calculate, it is preload dependent and does not correlate with the symptoms. Moreover, its specificity in detecting incipient changes in the myocardium is low. Kostam et al showed that routinely used techniques are observer dependent and have low sensitivity and specificity [12]. Speckle tracking echocardiography (STE) has overcome these limits, by allowing both global and regional analysis of myocardial deformation, in different directions and angle independent $[13,14]$.

In this context, the aim of this review is to underline the use of advanced echocardiographic parameters in identifying patients at risk for developing post-acute myocardial infarction heart failure and subsequent adverse events.

\section{Global strain of the $\mathbf{L V}$}

After STEMI and PCI, the injured myocardium may recover or may become irreversibly remodeled. Remodeling occurs in $30-35 \%$ of the patients despite PCI, since the changes in the myocardial geometry begin in the acute phase of ischemia, increasing post-AMI mortality $[15,16]$. STE provides detailed assessment of global and regional myocardial LV deformation, which may not be detectable with LVEF. Longitudinal, circumferential and radial strains are the three directions of myocardial deformation that can be evaluated by STE [17]. Nevertheless, global longitudinal strain (GLS) was mostly evaluated in studies and was proven to have both diagnostic and prognostic implications [18].

\section{GLS versus $L V E F$ in the prediction of remodeling and $\mathrm{HF}$ of AMI patients}

Studies have shown that GLS was superior to LVEF in detection of systolic dysfunction, by demonstrating a significant reduction in GLS in the absence of a correspondent reduction of the LVEF in patients with coronary artery disease and HFpEF $[19,20]$. This can be explained by geometrical factors, as LVEF can be constant for a large variation in shortening if other geometric factors compensate (increased wall thickness and reduced EDV can maintain normal LVEF despite reduced shortening) [21].

Myocardial deformation is linked to the longitudinally oriented fibers, located in the sub-endocardium, which are the most susceptible to ischemic changes. It was demonstrated in many studies that GLS detects longitudinal abnormalities in the absence of reduction in LVEF [22-25]. This is due to the fact that fibers located in the mid-wall, which are circumferentially oriented, may compensate in the case of reduction of the longitudinal mechanics in order to maintain LVEF normal. Studies showed that circumferential shortening contributes twice as much in maintaining LVEF as longitudinal shortening [21].

However, some studies demonstrated a reduction in both GLS and global circumferential strain (GCS) while LVEF was still normal. This was shown to appear in ventricles with thicker walls or smaller volume, where both longitudinal and circumferential shortening are less required to maintain a normal LVEF [19].

Recent studies have explored the prognostic value of GLS in post-AMI and PCI patients. Park et al demonstrated that GLS was an independent predictor of HF and cardiac death in 50 anterior AMI patients treated with primary PCI [26]. Antoni et al demonstrated in a study performed on 659 patients with AMI treated with primary PCI, GLS (>-15.1\%) and longitudinal strain rate (>-1.06 sec-1) were independently correlated with mortality [27]. Ersbøl et al showed that a reduced GLS could predict HF in AMI patients even in the presence of a normal LVEF [28]. Furthermore, it was demonstrated that reduced GLS $(>-14 \%)$ was an independent predictor of mortality, HF and cardiac death in the absence of reduced LVEF [29]. In a study performed on 576 AMI patients treated with 
PCI, GLS was also independently associated with mortality, HF and stroke, in spite of normal LVEF and LV volumes. The most severe adverse events were experienced by patients with a GLS $\geq-10 \%$ [30]. Lacalzada et al demonstrated that GLS $(>-12.46 \%)$ was a predictor of adverse remodeling and cardiac events in patients with AMI [31]. Moreover, Bastawy et al demonstrated that GLS $(>-12.5 \%)$ and LV torsion $\left(<9.5^{\circ}\right)$ early after anterior STEMI treated with primary PCI are independent predictors of six months LV remodeling [24].

Therefore, GLS was able to predict post-AMI remodeling and HF not only for anterior location, but also in any other location (fig 1).

Several studies evaluated the role of STE in the prediction of LV remodeling after STEMI or NSTEMI [25-36], but there was one report exclusively investigating the role of strain in patients with post AMI HFpEF. Hsiao et al showed that remodeling occurred in $28.9 \%$ of patients with AMI and preserved LVEF after PCI. The study also reported a new index, the injury longitudinal strain (InjLS) as the average strain of which segmental longitudinal strains $>-15 \%$, which was an independent predictor for remodeling in patients with $\mathrm{HFpEF}$ even after PCI [37].

Considering these results, GLS is able to identify patients at risk, who might benefit from antiremodeling therapies (angiotensin-converting enzyme inhibitors, aldosterone antagonists, beta blockers) independently of reduced LVEF and it might represent a selection criteria for future studies.

\section{$G C S$ and radial strain in the prediction of}

remodeling and $\mathrm{HF}$ of $A M I$ patients

On the other hand, the role of GCS and global radial strain were only evaluated in small studies. Peak circumferential strain (PCS) was also a predictor of adverse remodeling [34,37-39]. Bonioset al [38] showed the value of PCS as a predictor of adverse remodeling in apical segments of the myocardium. The VALIANT study, performed on 603 patients with post AMI LV dysfunction or HF, showed that both longitudinal and circumferential strain rate were independent predictors of outcomes after AMI. Moreover, only circumferential strain rate was a predictor of LV remodeling, whereas global and radial strain rate were not [36], suggesting that preserved circumferential function might prevent ventricular enlargement after AMI. In the case of post-AMI HFpEF, it is hypothesized that circumferential and radial strains are relatively preserved in the initial process in order to maintain LVEF. This could be the reason why no association was found between GCS or global radial strain and LV remodeling in post AMI patients with HFpEF in the study performed by Hsiao et al [37] (fig 2).

\section{Normal, stunned or infarcted?}

Furthermore, there is data suggesting that STE could distinguish between normal, stunned and infarcted myocardium. Bachner-Hinenzonet al (study performed on ten pigs) demonstrated that circumferential strain was able to predict which segments will eventually become infarcted and which will be stunned. Moreover, when stunning resolved, peak circumferential strain was an indicator of transmurality. Thus, CS detects ischemia and reperfusion, while RS only detects ischemia at the acute stage of MI [40].

\section{Transmural versus non-transmural AMI}

Measurement of GCS can differentiate between subendocardial and transmural infarction [41-46]. Chan et al demonstrated that circumferential strain was preserved, while longitudinal strain was reduced in subendocardial infarcts. On the other hand, both circumferential and longitudinal strain were reduced in transmural infarcts. There was no difference in radial strain [41]. Becker et al found that circumferential strain had the highest sensitivity and specificity in distinguishing transmural from non-transmural infarction [43]. Recently, a study using layer specific STE analysis, demonstrated that both circumferential and longitudinal strains of endocardial, mid myocardial and epicardial layer can distinguish infarcted from non-infarcted segments and differentiate non-transmural from transmural AMI [6].

\section{$G L S$ in the prediction of post-AMI ventricular arrhythmias}

Prediction and prevention of ventricular arrhythmias and sudden cardiac death early after AMI has been a challenge. Haugaa et al confirmed the role of myocardial deformation in the prediction of ventricular arrhythmias [47]. The Defibrillator in Acute Myocardial Infarction Trial could not demonstrate a survival advantage of implantable cardioverter defibrillator (ICD) implantation within 40 days in AMI patients with an LVEF $\leq 35 \%$ [48]. Ersbøl et al demonstrated additional information on longitudinal strain to LVEF in the early phase of AMI for identifying patients who might benefit from ICD implantation [49]. Therefore, GLS might be included next to LVEF in the LV evaluation of AMI patients.

\section{Regional strain of the LV}

Regional contraction is not only influenced by reduced contractility of the affected myocardium, but also by tension from the surrounding segments. Therefore, when ischemia occurs, shortening of the myocardial fibers decreases during LV ejection, and early systolic lengthening associated with post-systolic shortening can be observed in the ischemic myocardium. Strain param- 

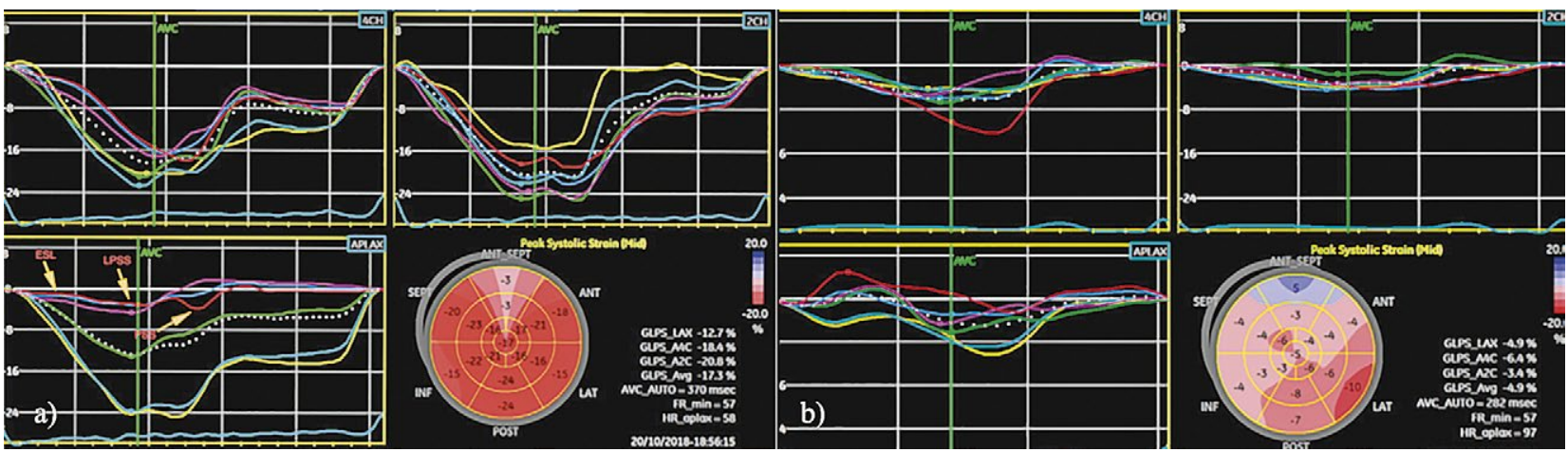

Fig 1. Left ventricular global longitudinal strain (GLS) in two post AMI patients: a) Calculated LVEF was 55\%. Left and right upper corner: curves derived from apical four-, three-, and two-chamber views show a reduced regional longitudinal strain in the anterior septal wall of a patient with LAD occlusion, 3 days after PCI. ESL, PSS and reduced LPSS can be noticed in the affected segments (red - basal, blue - mid, purple - apical segment of the anterior septum), with a normal aspect in the non-affected segments; right bottom: bull's eye plot with a mildly reduced GLS (-17.3\%) and visualization of the affected wall; b) Calculated LVEF was $45 \%$. Left and right upper corner: curves derived from apical four-, three-, and two-chamber views show a severely reduced regional longitudinal strain in the anterior, septal and inferior walls of a patient with anterior AMI 5 months after PCI and multi-vessel coronary artery disease. ESL and reduced LPSS can be noticed in the majority of the segments, while PSS is present in few segments (sign of chronic infarction); right bottom: bull's eye plot with significantly reduced GLS (-4.9\%) and visualization of the affected walls (ESL $=$ early systolic lengthening, PSS = post-systolic shortening, LPSS = longitudinal peak systolic strain, ESS = end-systolic strain).

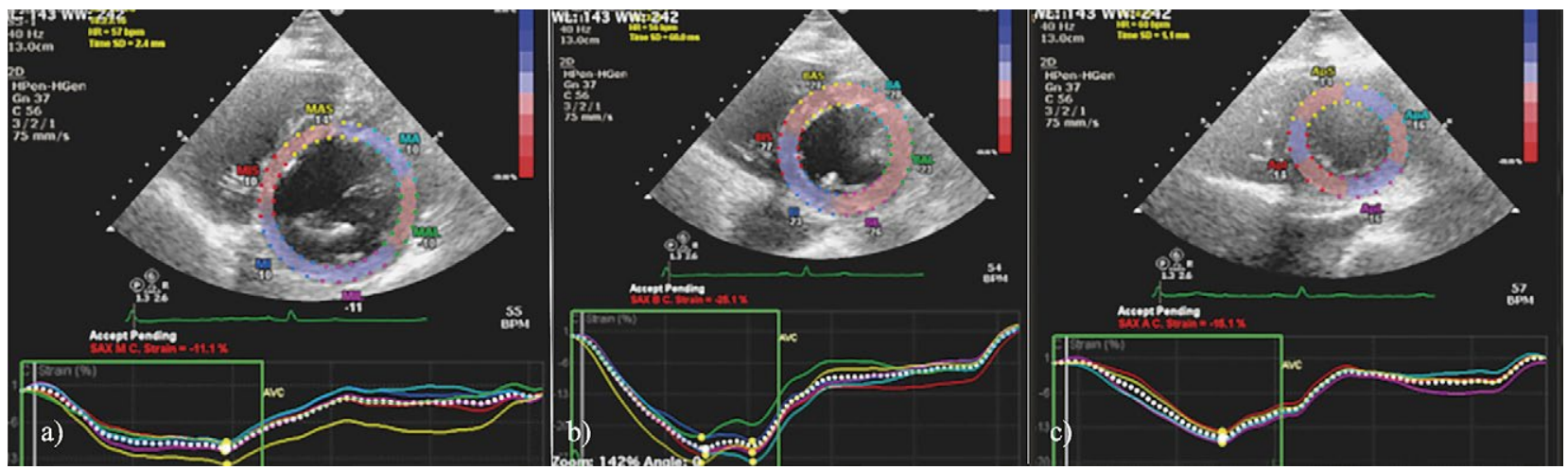

Fig 2. Mildly reduced regional circumferential strain (basal and apical segments) in a patient with anterior STEMI with distal left anterior descending coronary artery occlusion and multi-vessel coronary artery disease, 3 days after PCI: a) at the level of the mitral valve; b) at the level of the papillary muscles; c) at the level of the apex. LVEF was $52 \%$.
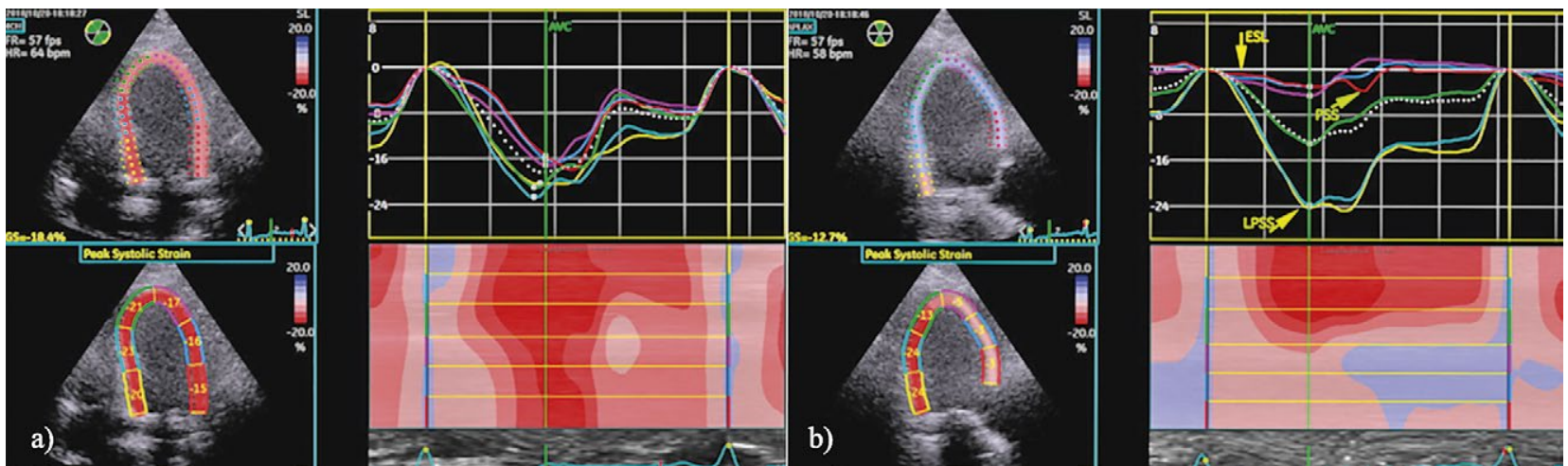

Fig 3. Regional longitudinal strain: a) curves derived from 4 chambers view in a normal patient; b) curves derived from 3 chambers view in a patient with anterior STEMI, 2 days after PCI. LPSS coincides with ESS in this case and is reduced in the affected segments. Moreover, ESL and PSS can be noticed in the segments with a reduced LPSS. LVEF was 55\% (ESL = early systolic lengthening, PSS = post-systolic shortening, LPSS = longitudinal peak systolic strain, ESS = end-systolic strain) 
eters can characterize ischemic dysfunction by showing reduction in the peak systolic strain, low systolic shortening, presence of systolic lengthening (SL) and post-systolic shortening (PSS) (fig 3).

\section{Longitudinal peak systolic strain (LPSS) and end- systolic strain}

Original Task Force recommendations in standardization of deformation suggested that end-systolic strain was able to evaluate systolic function more accurately than LPSS, due to the fact that peak systolic strain can show normal values when marked PSS is present in ischemic segments. Despite these findings, subsequent inter-vendor analysis reports recommended the use of LPSS in the evaluation of systolic dysfunction [50,51].

Kalam et al suggests that global LPSS (GLPSS) is superior to LVEF in detection of ischemia. The reason is the localization of longitudinal fibers in the sub-endocardial layer, which is the first one affected by ischemia [12]. Another study demonstrated that GLPSS is useful in the evaluation of transmuralityin the setting of a first AMI [36].

\section{Systolic lengthening (SL) and duration of the early} systolic lengthening (DESL)

Myocardium affected by ischemia tends to stretch during early systole before the onset of systolic shortening (when the pressure rises in the LV), because of its reduced ability in generating active force. After the acute event, viable myocardium progressively recovers, whereas necrotic segments preserve the same pattern of SL. DESL is a novel parameter, which reflects the time fibers remain stretched. Smedsrud et al demonstrated the superiority of DESL over GLPSS in identifying patients with significant coronary artery disease. A value of DESL $>58 \mathrm{~ms}$ had good sensitivity and specificity in detecting significant coronary artery disease [20,21]. DESL also correlated with the infarct size and was able to distinguish coronary occlusions from non-occlusions. Moreover, Zahid et al demonstrated that this parameter successfully identified NSTEMI patients with no visible scar on magnetic resonance [52].

Detection of post-AMI viable myocardium is a prognostic factor of remodeling and HF. When a myocardial segment has a $\mathrm{SL}>40 \%$ of systole there is a high probability of no recovery. Furthermore, when more than 4 segments show this pattern of prolonged SL, there is a high probability of no recovery of the LV function [53].

Post-systolic strain, post-systolic shortening (PSS) and post-systolic index (PSI)

Post-systolic strain and PSS have been proposed as markers of viability in some studies. PSS occurs in both viable and necrotic myocardium. In viable myocardium, it is attributed to the interaction between ischemic and sur- rounding myocardium, due to passive recoil. In the latter case, it is associated with SL and occurs when LV pressure is falling down during isovolumic relaxation [54].

Pislaru et al demonstrated that PSS parameters are the most specific in differentiating ischemic from non-ischemic ischemia during acute coronary occlusion [55]. On the other hand, it might be difficult to assess ischemic alterations in a previously damaged myocardium. Claus et al showed that PSS was not present in chronic infarction, suggesting that increased myocardial stiffness can diminish PSS caused by passive recoil [56].

What seems to remain an open issue is which direction of strain should be analyzed in order to assess PSS in STE. LS are the most frequently used, but the acquisition is less reliable in apical segments because of technical difficulties. This may interfere with the accuracy of diagnosing anterior AMI.

The post-systolic index represents the ratio between the amplitude of PSS to total shortening and it is calculated as (PSS-ESS)/peak strain or maximum strain change. It was shown to be effective in distinguishing ischemic from non-ischemic segments in association with PSS [57]. Furthermore, PSI increased significantly in graded coronary flow reduction, even when PSS did not decrease $[55,58]$. It still remains a question how to differentiate between physiologic and pathologic PSI. Moreover, the cut-off values of PSI are still unclear.

Tarkelsen et al concluded that PSS by tissue Doppler imaging was not a marker of viability in STEMI patients after revascularization [58]. Despite these findings, several other studies demonstrated that PSS was a marker of viability, and moreover a predictor of systolic recovery. Eek et al showed that PSS assessment before revascularization in NSTEMI patients predicted systolic recovery and HF [59]. The findings were confirmed by Hosokawa et al (in post-STEMI patients treated with PCI) [60] and Song et al (with stress echocardiography after AMI) [61]. Furthermore, Brainin et al found that PSS in the septal wall was associated with an increased risk of HF [54]. This is due to the fact that myocardial fibers in this zone suffer greatest deformation during systole and are exposed to the highest wall stress. PSS measured by STE was found to be an independent predictor of HF [62].

In conclusion speckle tracking echography represents a very useful, non-invasive method in the early diagnosis of LV remodeling after an acute myocardial infarction, with a high prognostic value in predicting subsequent HF development, which might be prevented with the early initiation of adequate therapy.

Conflict of interest: none 


\section{References}

1. Ibanez B, James S, Agewall S, et al. 2017 ESC Guidelines for the management of acute myocardial infarction in patients presenting with ST-segment elevation: The Task Force for the management of acute myocardial infarction in patients presenting with ST-segment elevation of the European Society of Cardiology (ESC). Eur Heart J 2018;39:119-177.

2. Azevedo PS, Polegato BF, Minicucci MF, Paiva SA, Zornoff LA. Cardiac Remodeling: Concepts, Clinical Impact, Pathophysiological Mechanisms and Pharmacologic Treatment. Arq Bras Cardiol 2016;106:62-69.

3. Chong J, Bulluck H, Yap EP, Ho AF, Boisvert WA, Hausenloy DJ. Remote ischemic conditioning in ST-segment elevation myocardial infarction - an update. Cond Med 2018;1:13-22.

4. Antonelli L, Katz M, Bacal F, et al. Heart Failure with Preserved Left Ventricular Ejection Fraction in Patients with Acute Myocardial Infarction. Arq Bras Cardiol 2015;105:145-50.

5. Yoon HJ, Kim KH, Kim JY, et al. Impaired diastolic recovery after acute myocardial infarction as a predictor of adverse events. J Cardiovasc Ultrasound 2015;23:150-157.

6. Gerber Y, Weston SA, Berardi C, et al. Contemporary trends in heart failure with reduced and preserved ejection fraction after myocardial infarction: a community study. Am J Epidemiol 2013;178:1272-1280.

7. Wdowiak-Okrojek K, Wejner-Mik P, Kasprzak JD, Lipiec P. Recovery of regional systolic and diastolic myocardial function after acute myocardial infarction evaluated by two-dimensional speckle tracking echocardiography. Clin Physiol Funct Imaging 2018 Nov 7. doi:10.1111/cpf.12553.

8. Ponikowski P, Voors AA, Anker SD, et al. 2016 ESC Guidelines for the diagnosis and treatment of acute and chronic heart failure: The Task Force for the diagnosis and treatment of acute and chronic heart failure of the European Society of Cardiology (ESC)Developed with the special contribution of the Heart Failure Association (HFA) of the ESC. Eur Heart J 2016;37:2129-2200.

9. Gho JMIH, Postema PG, Conijn M, et al. Heart failure following STEMI: a contemporary cohort study of incidence and prognostic factors. Open Heart 2017;4:e000551.

10. Lemor A, Hernandez GA, Patel N, et al. Predictors and etiologies of 30-day readmissions in patients with non-STelevation acute coronary syndrome. Catheter Cardiovasc Interv 2018 Oct 2. doi:10.1002/ccd.27838.

11. Hendricks T, Hartman MHT, Vlaar PJJ, et al. Predictors of left ventricular remodeling after ST-elevation myocardial infarction. Int J Cardiovasc Imaging 2017;33:1415-1423.

12. Konstam MA, Kramer DG, Patel AR, Maron MS, Udelson JE. Left ventricular remodeling in heart failure: current concepts in clinical significance and assessment. JACC Cardiovasc Imaging 2011;4:98-108.

13. Hsiao JF, Chung CM, Chu CM, et al. Two-Dimensional Speckle Tracking Echocardiography Predict Left Ventricular Remodeling after Acute Myocardial Infarction in Patients with Preserved Ejection Fraction. PloS One 2016;11:e0168109.

14. Mele D, Luisi GA, Pestelli G, Smarrazzo V, Trevisan F, Ferrari R. Echocardiography in ST-elevation myocardial infarction. G Ital Cardiol 2018;19:479-487.

15. Karabağ Y, Çınar T, Çağdaş M, Rencüzoğulları İ, Tanık VO. In-hospital and long-term prognoses of patients with a mid-range ejection fraction after an ST-segment myocardial infarction. Acta Cardiol 2018 Aug 23:1-8. doi:10.1080/000 15385.2018.1501140.

16. Ola RK, Meena CB, Ramakrishnan S, Agarwal A, Bhargava S. Detection of Left Ventricular Remodeling in Acute ST Elevation Myocardial Infarction after Primary Percutaneous Coronary Intervention by Two Dimensional and Three Dimensional Echocardiography. J Cardiovasc Echogr 2018;28:39-44.

17. Voigt JU, Pedrizzetti G, Lysyansky P, et al. Definitions for a commonstandard for 2D speckle tracking echocardiography: consensus document of theEACVI/ASE/Industry Task Force to standardize deformation imaging. Eur Heart J Cardiovasc Imaging 2015;16:1-11.

18. Kalam K, Otahal P, Marwick TH. Prognostic implications of global LV dysfunction:a systematic review and metaanalysis of global longitudinal strain and ejection fraction. Heart 2014;100:1673-1680.

19. Kraigher-Krainer E, Shah AM, Gupta DK, et al, Impaired systolic function by strain imaging in heart failure with preserved ejection fraction. J Am Coll Cardiol 2014;63:447456.

20. Smedsrud MK, Sarvari S, Haugaa KH, et al. Duration of myocardial early systolic lengthening predicts the presence of significant coronary artery disease. J Am Coll Cardiol 2012;60:1086-1093.

21. Stokke TM, Hasselberg NE, Smedsrud MK, et al. Geometry as a Confounder When Assessing Ventricular Systolic Function, Comparison Between Ejection Fraction and Strain. J Am Coll Cardiol 2017;70:942-954.

22. Mor-Avi V, Lang RM, Badano LP, et al. Current and evolving echocardiographic techniques for the quantitative evaluation of cardiac mechanics: ASE/EAE consensus statement on methodology and indications. Eur J Echocardiogr 2011;12:167-205.

23. Prastaro M, Pirozzi E, Gaibazzi N, et al. Expert Review on the Prognostic Role of Echocardiography after Acute Myocardial Infarction. J Am Soc Echocardiogr 2017;30:431443.e2.

24. Bastawy I, Ismail M, Hanna HF, Kilany WE. Speckle tracking imaging as a predictor of left ventricular remodeling 6 months after first anterior ST elevation myocardial infarction in patients managed by primary percutaneous coronary intervention. Egypt Heart J 201817 July. doi:10.1016/j. ehj.2018.06.006.

25. Ismail AM, Samy W, Aly R, Fawzy S, Hussein K. Longitudinal strain in patients with STEMI using speckle tracking echocardiography. Correlation with peak infarction mass and ejection fraction. The Egyptian Journal of Critical Care Medicine 2015;3:45-53. 
26. Park YH, Kang SJ, Song JK, et al. Prognostic value of longitudinal strain after primary reperfusion therapy in patients with anterior-wall acute myocardial infarction. J Am Soc Echocardiogr 2008;21:262-267.

27. Antoni ML, Mollema SA, Delgado V, et al. Prognostic importance of strain and strain rate after acute myocardial infarction. Eur Heart J 2010;31:1640-1647.

28. Ersbøll M, Valeur N, Mogensen UM, et al. Relationship between left ventricular longitudinal deformation and clinical heart failure during admission for acute myocardial infarction: a two-dimensional speckle-tracking study. J Am Soc Echocardiogr 2012;25:1280-1289.

29. Ersbøll M, Valeur N, Mogensen UM, et al. Prediction of all-cause mortality and heart failure admissions from global left ventricular longitudinal strain in patients with acute myocardial infarction and preserved left ventricular ejection fraction. J Am Coll Cardiol 2013;61:2365-2373.

30. Munk K, Andersen NH, Terkelsen CJ, et al. Global left ventricular longitudinal systolic strain for early risk assessment in patients with acute myocardial infarction treated with primary percutaneous intervention. J Am Soc Echocardiogr 2012;25:644-651.

31. Lacalzada J, de la Rosa A, Izquierdo MM, et al. Left ventricular global longitudinal strain predicts adverse remodeling and subsequent cardiac events in patients with acute myocardial infarction treated with primary percutaneous intervention. Int J Cardiovasc Imaging 2015;31:575-584.

32. Keddeas VW, Swelim SM, Selim GK. Role of 2D speckle tracking echocardiography in predicting acute coronary occlusion in patients with non ST-segment elevation myocardial infarction. Egypt Heart J 2017;69:103-110.

33. Wdowiak-Okrojek K, Wejner-Mik P, Kasprzak JD, Lipiec P. Recovery of regional systolic and diastolic myocardial function after acute myocardial infarction evaluated by two-dimensional speckle tracking echocardiography. Clin Physiol Funct Imaging 2018 Nov 7. doi:10.1111/cpf.12553.

34. Altiok E, Tiemann S, Becker M, et al. Myocardial deformation imaging by two-dimensional speckle-tracking echocardiography for prediction of global and segmental functional changes after acute myocardial infarction: a comparison with late gadolinium enhancement cardiac magnetic resonance. J Am Soc Echocardiogr 2014;27:249-257.

35. Joyce E, Hoogslag GE, Leong DP, et al. Association between left ventricular global longitudinal strain and adverse left ventricular dilatation after ST-segment-elevation myocardial infarction. Circ Cardiovasc Imaging 2014;7:74-81.

36. Mangion K, Carrick D, Carberry J, et al. Circumferential Strain Predicts Major Adverse Cardiovascular Events Following an Acute ST-Segment-Elevation Myocardial Infarction. Radiology 2018 Nov 20. doi:10.1148/radiol.2018181253.

37. Bendary A, Afifi M, Tawfik W, Mahros M, Ramzy A, Salem $M$. The predictive value of global longitudinal strain on late infarct size in patients with anterior ST-segment elevation myocardial infarction treated with a primary percutaneous coronary intervention. Int J Cardiovasc Imaging 2018 Nov 14. doi:10.1007/s10554-018-1498-7.
38. Bonios MJ, Kaladaridou A, Tasoulis A, et al. Value of apical circumferential strain in the early post-myocardial infarction period for prediction of left ventricular remodeling. Hellenic J Cardiol 2014;55:305-312.

39. Schuster A, Backhaus SJ, Stiermaier T, Eitel I. Prognostic utility of global longitudinal strain in myocardial infarction. World J Cardiol 2018;10:35-37.

40. Bachner-Hinenzon N, Malka, A, Barac, Y, et al. Strain Analysis in the Detection of Myocardial Infarction at the Acute and Chronic Stages. Echocardiography 2016;33:450458.

41. Chan J, Hanekom L, Wong C, Leano R, Cho GY, Marwick TH. Differentiation of Subendocardial and Transmural Infarction Using Two-Dimensional Strain Rate Imaging to Assess Short-Axis and Long-Axis Myocardial Function. J Am Coll Cardiol 2006;48:2026-2033.

42. Sugano A, Seo Y, Ishizu T, et al. Value of 3-Dimensional Speckle Tracking Echocardiography in the Prediction of Microvascular Obstruction and Left Ventricular Remodeling in Patients With ST-Elevation Myocardial Infarction. Circ J 2017;81:353-360.

43. Luo Y, Liu Y, Guan X, Zhang Y, Li J. Value of three dimensional-speckle tracking imaging for predicting left ventricular function after non-ST-segment elevation myocardial infarction with percutaneous coronary intervention. J Xray Sci Technol 2018;26:331-339.

44. Rost C, Rost MC, Breithardt OA, et al. Relation of functional echocardiography parameters to infarct scar transmurality by magnetic resonance imaging. J Am Soc Echocardiogr 2014;27:767-774.

45. Tsadok Y, Friedman Z, Haluska BA, Hoffmann R, Adam D. Myocardial strain assessment by cine cardiac magnetic resonance imaging using non-rigid registration. Magn Reson Imaging 2014;34:381-390.

46. Qiao Z, Zhao H, Sun JP, et al. Assessment of Acute Infarct Transmurality on Layer-Specific Impairment of Myocardial Function Using Myocardial Deformation Imaging and Compared with Contrast-Enhanced Cardiac Magnetic Resonance. Int Phys Med Rehab J 2017;1:00034.

47. Haugaa KH, Grenne BL, Eek CH, et al. Strain echocardiography improves risk prediction of ventricular arrhythmias after myocardial infarction. JACC Cardiovasc Imaging 2013;6:841-850.

48. Priori SG, Blomstroem-Lundqvist C, Mazzanti A, et al. 2015 ESC guidelines for the management of patients with ventricular arrhythmias and the prevention of sudden cardiac death: the Task Force for the Management of Patients with Ventricular Arrhythmias and the Prevention of Sudden Cardiac Death of the European Society of Cardiology (ESC). Endorsed by: Association for European Paediatric and Congenital Cardiology (AEPC). Eur Heart J 2015;36:2793-2867.

49. Ersbøll M, Valeur N, Andersen MJ, et al. Early echocardiographic deformation analysis for the prediction of sudden cardiac death and life-threatening arrhythmias after myocardial infarction. JACC Cardiovasc Imaging 2013;6:851860 . 
50. Collier P, Phelan D, Klein A. A Test in Context: Myocardial strain Measured by Speckle Tracking Echocardiography. J Am Coll Cardiol 2017;69:1043-1056.

51. Farsalinos KE, Daraban AM, Unlu S, et al. Head-tohead comparison of global longitudinal strain measurements among nine different vendors: the EACVI/ASE Inter-Vendor Comparison study. J Am Soc Echocardiogr 2015;28:1171-1181.e2.

52. Zahid W, Eek CH, Remme EW, Skulstad H, Fosse E, Edvardsen T. Early systolic lengthening may identify minimal myocardial damage in patients with non-ST-elevation acute coronary syndrome. Eur Heart J Cardiovasc Imaging 2014;15:1152-1160.

53. Meimoun, P, Abouth S, Clerc J. Usefulness of two-dimensional longitudinal strain pattern to predict left ventricular recovery and in-hospital complications after acute anterior myocardial infarction treated successfully by primary angioplasty. J Am Soc Echocardiogr 2015;28;1366-1375.

54. Brainin P, Haahr-Pedersen S, Sengeløv M, et al. Presence of post-systolic shortening is an independent predictor of heart failure in patients following ST-segment elevation myocardial infarction. Int J Cardiovasc Imaging 2018;34:751-760.

55. Pislaru C, Anagnostopoulos PC, Seward JB, Greenleaf $\mathrm{JF}$, Belohlavek M. Higher myocardial strain rates during isovolumic relaxation phase than during ejection characterize acutely ischemic myocardium. J Am Coll Cardiol 2002;40:1487-1494.
56. Claus P, Weidemann F, Dommke C, et al. Mechanisms of postsystolic thickening in ischemic myocardium: mathematical modelling and comparison with experimental ischemic substrates. Ultrasound Med Biol 2007;33;1963-1970.

57. Masuda K, Asanuma T, Taniguchi A, Uranishi A, Ishikura F, Beppu S. Assessment of dyssynchronous wall motion during acute myocardial ischemia using velocity vector imaging. JACC Cardiovasc Imaging 2008;1:210-220.

58. Terkelsen CJ, Poulsen SH, Nørgaard BL, et al. Does postsystolic motion or shortening predict recovery of myocardial function after primary percutanous coronary intervention? J Am Soc Echocardiogr 2007;20:505-511.

59. Eek C, Grenne B, Brunvand H, et al. Postsystolic shortening is a strong predictor of recovery of systolic function in patients with non-ST-elevation myocardial infarction. Eur J Echocardiogr 2011;12:483-489.

60. Hosokawa H, Sheehan FH, Suzuki T. Measurement of postsystolic shortening to assess viability and predict recovery of left ventricular function after acute myocardial infarction. J Am Coll Cardiol 2000;35:1842-1849.

61. Song JK, Song JM, Kang DH, Haluska B, Marwick TH. Postsystolic thickening detected by Doppler myocardial imaging: a marker of viability or ischemia in patients with myocardial infarction. Clin Cardiol 2004;27:29-32.

62. Smiseth OA, Torp H, Opdahl A, Haugaa KH, Urheim S. Myocardial strain: how useful is it in clinical decision making. Eur Heart J 2016;37:1196-1207. 